After a sufficiently great number of crossings and re-crossings across the line $\mathrm{X}^{\prime} \mathrm{OX}$, the particle will cross this line very nearly at right angles, at some point, $N^{\prime}$. Vary the position of $N$ very slightly in one direction or other, and re-project $m$ from it perpendicularly and with proper velocity; till (by proper "trial and error" method) a path is found, which, after still the same number of crossings and re-crossings, crosses exactly at right angles at a point $\mathrm{N}^{\prime \prime}$, very near the point $\mathrm{N}^{\prime}$. Let $m$ continue its journey along this path, and, after just as many more crossings and re-crossings, it will return exactly to $\mathrm{N}$, and cross $\mathrm{OX}$ there, exactly at right angles. Thus the path from $\mathrm{N}$ to $\mathrm{N}^{\prime \prime}$ is exactly half an orbit, and from $\mathrm{N}^{\prime \prime}$ to $\mathrm{N}$ the remaining half.

(I4) When $c \mathrm{E} /\left(\alpha^{2} \beta^{2}\right)$ is a small numeric, the part of the kinetic energy expressed by $\frac{1}{2} c x^{2} y^{2}$ is very small in comparison with the total energy, E. Hence the path is at every time very nearly the resultant of the two primary fundamental modes formulated in $\S \mathrm{I} 3$; and an interesting problem is presented, to find (by the method of the "variation of parameters") $a, e, b, f$, slowly varying functions of $t$, such that

$$
\begin{array}{ll}
x=a \sin (\alpha t-e), & y=b \sin (\beta t-f), \\
\dot{x}=\alpha \alpha \cos (\alpha t-e), & \dot{y}=b \beta \cos (\beta t-f),
\end{array}
$$

shall be the rigorous solution, or a practical approximation to it. Careful consideration of possibilities in respect to this case $\left[c \mathrm{E} /\left(\alpha^{2} \beta^{2}\right)\right.$ very small] seems thoroughly to confirm Maxwell's fundamental assumption quoted in $\$$ II ; and that it is correct whether $c \mathrm{E} /\left(\alpha^{2} \beta^{2}\right)$ be small or large seems exceedingly probable, or quite certain.

(I5) But it seems also probable that Maxwell's conclusion, which for the case of a material point moving in a plane is

$$
\text { Time-av, } \dot{x}^{2}=\text { Time-av. } \dot{y}^{2}, \ldots . . .
$$

is not true when $\alpha^{2}$ differs from $\beta^{2}$. It is certainly not proved. No dynamical principle except the equation of energy,

$$
\frac{1}{2}\left(\dot{x}^{2}+\dot{y}^{2}\right)=\mathrm{E}-\mathrm{V}
$$

is brought into the mathematical work of pp. $722-25$, which is given by curve might be assumed for the path without violating the dynamics which enters into Maxwell's investigation; and we may draw curves for the path such as to satisfy (I), and curves not satisfying (I), but all traversing the whole space within the bounding curve

$$
\frac{1}{2}\left(\alpha^{2} x^{2}+\beta^{2} y^{2}+c x^{2} y^{2}\right)=\mathrm{E},
$$

and all satisfying Maxwell's fundamental assumption (§ I I).

(I6) The meaning of the question is illustrated by reducing it to a purely geometrical question regarding the path, thus :Calling $\theta$ the inclination to $x$ of the tangent to the path at any point $x y$, and $q$ the velocity in the path, we have

$$
\dot{x}=q \cos \theta, \quad \dot{y}=q \sin \theta,
$$

and therefore, by (2),

$$
q=\sqrt{ }\{2(\mathrm{E}-\mathrm{V})\}
$$

Hence, if we call $s$ the total length of curve travelled,

$$
\int \dot{x}^{2} d t=\int q \cos ^{2} \theta q d t=\int \sqrt{ }\{2(\mathrm{E}-\mathrm{V})\} \cos ^{2} \theta d s ;
$$

and the question of $\S \mathrm{I} 5$ becomes, Is or is not

$$
\begin{aligned}
\frac{\mathbf{I}}{\mathrm{S}} \int_{0}^{s} d s \sqrt{ }\{2(\mathrm{E}-\mathrm{V})\} & \cos ^{2} \theta \\
= & \frac{\mathbf{I}}{\mathrm{S}} \int_{0}^{s} d s \sqrt{ }\{2(\mathrm{E}-\mathrm{V})\} \sin ^{2} \theta ? .
\end{aligned}
$$

where $\mathrm{S}$ denotes so great a length of path that it has passed a great number of times very near to every point within the boundary (3), very nearly in every direction.

(I 7$)$ Consider now separately the parts of the two members of (7) derived from portions of the path which cross an infinitesimal area $d \sigma$ having its centre at $(x, y)$. They are respectively

and

$$
\begin{aligned}
& \sqrt{ }\{2(\mathrm{E}-\mathrm{V})\} d \sigma \int_{0}^{\pi} \mathrm{N} d \theta \cos ^{2} \theta \\
& \sqrt{ }\{2(\mathrm{E}-\mathrm{V})\} d \sigma \int_{0}^{\pi} \mathrm{N} d \theta \sin ^{2} \theta
\end{aligned}
$$

where $\mathrm{N} d \theta$ denotes the number of portions of the path, per unit distance in the direction inclined $\frac{1}{2} \pi+\theta$ to $x$, which pass eitherwards across the area in directions inclined to $x$ at angles between the values $\theta-\frac{1}{2} d \theta$ and $\theta+\frac{1}{2} d \theta$. The most general possible expression for $\mathrm{N}$ is, according to Fourier,

$$
\left.\begin{array}{rl}
N=A_{0} & +A_{1} \cos 2 \theta+A_{2} \cos 4 \theta+\& c . \\
& +B_{1} \sin 2 \theta+B_{2} \sin 4 \theta+\& c .
\end{array}\right\} .
$$

Hence the two members of $(8)$ become respectively

and

$$
\left.\begin{array}{l}
\sqrt{ }\{2(\mathrm{E}-\mathrm{V})\} d \sigma \frac{1}{2} \pi\left(\mathrm{A}_{0}+\frac{1}{2} \mathrm{~A}_{1}\right) \\
\sqrt{ }\{2(\mathrm{E}-\mathrm{V})\} d \sigma \frac{1}{2} \pi\left(\mathrm{A}_{0}-\frac{1}{2} \mathrm{~A}_{1}\right)
\end{array}\right\} .
$$

Remarking that $\mathrm{A}_{0}$ and $\mathrm{A}_{1}$ are functions of $x, y$, and taking $d r=d x d y$, we find, from (IO), for the two lotals of (7) respectively

and

$$
\begin{aligned}
& \frac{1}{2} \pi \iint d x d y\left(\mathrm{~A}_{0}+\frac{1}{2} \mathrm{~A}_{1}\right) \sqrt{[2(\mathrm{E}-\mathrm{V})]} \mid, \ldots \text { (II) } \\
& \left.\frac{1}{2} \pi \iint d x d y\left(\mathrm{~A}_{0}-\frac{1}{2} \mathrm{~A}_{1}\right) \sqrt{ }[2(\mathrm{E}-\mathrm{V})]\right\}
\end{aligned}
$$

where $\iint d x d y$ denotes integration over the whole space inclosed by (3). These quantities are equal if and only if $\iint d x d y \mathrm{~A}_{1}$ vanishes; it does so, clearly, if $\alpha=\beta$; but it seems improbable that, except when $\alpha=\beta$, it can vanish generally; and unless it does so, our present test case would disprove the Boltzmann-Maxwell general doctrine.

\section{THE INTERNATIONAL GEOGRAPHICAL CONGRESS AT BERNE.}

THIS Congress began its proceedings on Monday. Fourteen countries and forty-six Geographical Societies are officially represented. France has sent 73 delegates, Germany 33, Austria-Hungary 2r, Swilzerland 87, Italy 21, Russia 13, Great Britain 8, and Spain, America, and the Netherlands two each. Egypt, Portugal, Roumania, Greece, Norway, and Sweden are also represented. There are, in addition, 150 Members and Associates who have not yet given in their names.

M. Numa Droz, Swiss Minister for Foreign Affairs, bade the delegates heartily welcome to Berne.

Dr. Gobat, Regierungsrath, Berne, President of the Congress, then delivered his inaugural address. In the name of the Geographical Societies of Switzerland he thanked the savants present for responding so cordially to their invitation.

Among the good work already done, Prof. Penck, of Vienna, has proposed the following resolution:- "This Congress on the geographical sciences, held at Berne, resolves to take the initiative in the preparation of a large map of the earth on a scale of one to a million, of which the various sections shall be de. limited by latitudes and longitudes; and, with this object, it appoints an international committee to determine the principles upon which the preparation of such map shall proceed. The members of this committee shall arrange that the various States engaged in preparing maps, the societies and periodicals pub. lishing original maps, and all private geographical establishments working in this field shall prepare detached sections of the said map, the sale of which shall also be regulated and arranged for by the committee."

In the course of his address on the subject Prof. Penck paid a high tribute to the services rendered by Mr. Stanley to the cause of geographical science, directing special attention to the fact that each of the explorer's expeditions across Africa had led to the preparation of from 20 to 30 maps.

The proposal was referred to a committee of the Congress, which will report upon it.

The subjects of an initial meridian and universal time, geographical education, orthography of geographical names, lakes and glaciers, cartography, bibliography, meteorology, commercial geography, and voyages and travels are all to be touched upon in the deliberations.

\section{SCIENTIFIC SERIAISS.}

Joumal of the Russian Chemical and Physical Society, vol. xxiii., No. 1. - The chief papers are :-On the molecular weight of albumen, by A. Sabanéeff and N. Alexandroff. Several determinations were made on the method of Raoult, and gave an average of 14,276 , the molecular weight thus appearing to be nearly three times as great as that deduced from the formula of

NO. I I 37 , VOL. 44] 